\title{
A CASE STUDY ON CPE (COMMUNICATION PROTOCOL ERROR) ALARM IN NOKIA DXC
}

\author{
Rupinder Kaur ${ }^{1}$, Vimal Kant Kanaujia ${ }^{2} \&$ Harpreet Singh $^{3}$ \\ Abstract-The case study is on CPE (Communication Protocol Error) issue in Nokia DXC (Digital Cross-Connect) that caused \\ traffic outage in network. The case study involves the description of technology (SDH- Synchronous Digital Hierarchy), \\ Protection schemes and product which would help understanding the issue details and the reasons for which the issue occurred. \\ Moreover, the bytes K1 K2 are responsible for switching the stream of traffic from main to protection path when failure occurs \\ in the main path of a section in a ring. Therefore, to understand the purpose of $\mathrm{K} 1 \mathrm{~K} 2 \mathrm{bytes}$, the case study involves the full \\ description of the technology supported by the Nokia product DXC. \\ Keywords - SDH, MS-SPRing, Switching, K1 K2 bytes, Main path, Protection path
}

\section{INTRODUCTION}

In the last years of 1960s and early 1970s plesiochronous multiplexing was used that provided 140Mbit/s data rate. With the growing demands and increasing digital network complexity, the network operators were pressurized to raise network features improving functionality of the data transfer rate. With the introduction of optical fibers, the new multiplexing method known as SDH was introduced. Earlier the standard was known as SONET (Synchronous Optical Networking). Later, ANSI limited SONET standards for use in North America only and SDH was defined by ETSI (a subset of ITU-T) for the use in the rest of the world. [1]

PDH provided asynchronous digital hierarchy therefore introduction of SDH in 1988 lead to solution of timing problem of PDH systems. When asynchronous PDH signals are fed to the SDH multiplexer, multiple VCs (Virtual Containers) which are timed by different clocks combines into one STN-N signal that the local equipment clock times. [2] SDH uses STM-1 frame as the basic frame for transmitting signals which can be byte interleaved to form higher rate STM- N signal. The basic frame has $155.52 \mathrm{Mbit} / \mathrm{s}$ bit rate which is made up of nine rows by 270 columns each of eight -bit bytes. Thus, forming 155.52 Mbit/s bit rate as $270 * 9=2430$ bytes. The transmission of these many bytes have to take place within 125 microseconds. [3] SDH data structure uses multiples of STM-1 frame with the lowest granularity channel of 2 Mbit/s. This has ease the option of using a single equipment for adding, removing or switching this lowest channel of 2 Mbit/s to any STM-N level directly. For low populated locations, STM-1 frame is sufficient but for densely populated/business areas, STM-4 or STM-16 is required. [4]

\section{LITERATURE SURVEY}

The authors have analyzed two protection ways in their paper, one is Path protection and second one is self-healing ring with two fiber and four fiber. They also analyzed how $2 \mathrm{Mbps}$ signal is available between two nodes using the two mentioned protection schemes. For path protection, always the higher quality signal is chosen by the receiver side. For self-healing rings, they explain $\mathrm{K} 1 \mathrm{~K} 2$ bytes come into action to reroute the signal to the spare path when the network fails. [5]

The SDH/ SONET standards have the same explanation of switching to protection paths in the instance of failure of main path. The switching depends on K1K2 bytes which are present in Multiplexer Section (MSOH) of the SDH frame. The receiving end generates the switching command when failure occurs so that the traffic could be switched onto the protection path. The comparison of protection logic at receiving end takes place as per the request of received K1 byte. The comparison of the new request is done with the old one and if it is of higher priority then byte $\mathrm{K} 1$ at receiving/tail end is loaded with the new request. Also, the ID of the channel that is to be switched to, is loaded into the byte K1. The tail end then sends the K1 byte through protection line. The head end when receives this new K1 byte through protection line, sends back the reverse request to the tail end to confirm that the protection path is ready to be used as the medium of communication. The abovementioned process allows the swapping of head end and a K2 byte with the channel ID is sent to the tail end. Thus, switching occurs at tail end as per its $\mathrm{K} 1$ byte and head end has to be sent the K2 byte.

Finally switching process is completed when head end receives the matching K2 byte from the tail end. The switching process must take place within $50 \mathrm{msec}$ for effective transmission of data frames in the network. [6,7]

\footnotetext{
${ }^{1}$ Department of Computer Science and Engineering, ME Scholar, Chandigarh University, Mohali, Punjab

${ }^{2}$ Senior Engineering Manager IPTNEGTAC, Nokia, Gurgaon

${ }^{3}$ Department of Computer Science and Engineering, Assistant Professor, Chandigarh University, Mohali, Punjab
} 
In this paper, the author discuss that SDH network can be extended further to build multiservice networks. It depends on the city size and traffic quantity; the network stream can vary from STM-1 to STM-N. Also, they discuss the optimization of SNCP and MS-SPRing protection methods. After carrying out cost analysis MS-SPRing protection method is better and efficient than SNCP method. [8]

The author of the paper defines the use of the technology SDH along with Fiber-in-the-loop (FITL) in Deutsche Telecom (DT). They have also mentioned that this technology is efficient enough to handle the requirements of great sum of customers fulfilling their needs of additional services. SMT or Synchronous Multiplexer Terminal is interesting for use by broadband services as it provides the $155 \mathrm{Mb} / \mathrm{s}$ data rate. [9]

The authors of this paper have assessed the availability networks using SDH to discuss the facts causing the network outages and for how long the services are affected. It is necessary to analyze whether the network is efficient enough to complete customer needs. The authors conclude that the network's performance measurement would help the network operators to focus on the reason for outages and another network effecting defect which would be helpful to prevent the traffic impacts in future. [10]

The authors in [11] tells that protection can either be $1+1$ or $1: \mathrm{N}$ which means that for $1+1$ protection there is a devoted protection channel for working channels and for 1: $\mathrm{N}$ protection, it's only a single protection channel shared by working channels $\mathrm{N}$ in number. The switching type can either be unidirectional or bidirectional and revertive or non-revertive. For unidirectional, if one side of fiber fails, then only that side will switch to its protection, the other half of pair will continue transmission and receival using its working channel. For bidirectional, if there is failure in any of the fiber pair, the whole pair will switch to the channel defined as protection path. Revertive means that after the restoration of main working channel the traffic switch back from protection to the working channel whereas in non-revertive switching, the traffic will continue movement in the channel to which it is switched to.

In [12] the author has discussed a protocol needed to maintain communication between the working channel interface and the standby interface. In normal conditions both the channels share hello messages so that keep-alive status is maintained between the two. Typical settings of the router involve sharing of three messages per second. When these three messages are absent, it indicates outage to the standby path. These are default settings which vary from vendor to vendor.

The authors of this paper describe that the transmission system based on SDH technology are very efficient for multiplexing of signals at different speed into desired speed signal. It is easy to access any tributary and any channel in a signal that is multiplexed using SDH technology. [13] The multiplexing schemes are of two types- the first one being byte multiplexing or byte interleaving and the second one is bit multiplexing or bit interleaving. The paper describes that the SDH systems deployed in network suitably works with byte interleaving scheme as per ITU-T recommendations. [14]

The ring structure behaves to be the simple topology implemented to provide easy control as well as fast restoration in the communication network. The paper emphases on the description of $2 \mathrm{~F}$ and $4 \mathrm{~F}$ self-healing rings which are amongst the most used ring structures in SDH network. Thus, the authors analyze how well the network survives during failures and how the protection scheme works in that case. [15] The paper gives an overview how a PDH tributary (2.048 Mbit/s) asynchronous in nature is mapped into SDH VC-12. It describes that a synchronizer is present that provides acceptance of the PDH tributary into SDH network and it maps it with the SDH floating VC-12 payload structure. SDH frame is used to take the PDH tributary into network. [16]

In [17] there is the description of three optical layers, OCH, OMS, OTS where OCH is the layer of optical channel (SDH, PDH and ATM) providing end to end connection in the network for efficient communication amongst clients. The second layer being OMS (optical multiplex section) provides functionality to the network and the layer three, OTS (optical transmission section) is responsible for transmission of optical signals.

The authors discuss in their paper that Ethernet has no functionality of protection switching whereas SONET and SDH frames has this function of protection switching that is done using K1 K2 bytes which increases the network efficiency based on these technologies. There are overhead bytes provided by the frame structure of SONET/SDH that provides fault isolation. [18] The growing needs of people from telecommunication world has pressurized the telecommunication world to advance their environments to support high speed demand. This paper describes a BDCS (Broadband DCS) that supports SDH technology to meet up demand of people. [19]

ITU-T Rec. G.841 describes that legacy SONET/SDH ring networks are good at providing protection switching functionality within the time of 50 milli sec whenever the fault is detected. [20] In [21] it is described that the media issues can occur due to digging work or it may be due to failure of one or greater than one transmitters or receivers. Therefore, the failure in optical layer adversely effects the network performance. The protection schemes that are required when failure occurs is one of the two- dedicated or shared. Out of these two the protection system of shared type is commonly used in networks as it uses less resources but its implementation is difficult and complex than the dedicated approach of protection. [22]

The article discusses the application of new and old switching technologies that could serve all protection following applications. The authors discuss the probability of network survivability to be completely dependent on recovery from failures. Recovery of fault involves two mechanisms protection and restoration. [23] The paper confers that SDH technology being initially deployed had to provide quality service to the customer both when the network is running smoothly and the conditions of some fault in network or network failure occurs. [24] 
The advancement of technologies in the field of optical networking does not put an end to the existing technologies like SDH. If it is proposed not to use SDH for switching purpose still its frame should serve the switch port. Moreover, if the fibers are connected directly, it would be efficient only for short distances; the SDH network supports the management and utilization of fibers efficiently [25] The SDH networks will not diminish but they are likely to be soon acquired by WDMs as switching is more economical if done directly or using WDM (Wavelength Division Multiplexing) devices. [26]

\section{DESCRIPTION OF TECHNOLOGY USED}

The technology used here is SDH or Synchronous Digital Hierarchy. The network element mentioned in the paper uses this technology. The basic frame of this technology is STM-1 as figure 1 shows. The frame size is 270 columns by 9 rows and STM-1 frame has 155.520Mbps bit rate.

Table 1: STM-n bit rate (Source:27)

\begin{tabular}{|l|l|}
\hline STM frame & Bit Rate \\
\hline STM-1 & $155.52 \mathrm{Mbit} / \mathrm{s}$ \\
\hline STM-4 & $622.08 \mathrm{Mbit} / \mathrm{s}$ \\
\hline STM-16 & $2588.32 \mathrm{Mbit} / \mathrm{s}$ \\
\hline STM-64 & $9953.28 \mathrm{Mbit} / \mathrm{s}$ \\
\hline
\end{tabular}

We can see in Figure 1 that STM-1 frame is made up of two parts-

- Section Overhead: The first nine columns of the frame forms section overhead forming 81 bytes' section overhead.

- Virtual container: The next 261 columns forms the virtual container of 2349 bytes.

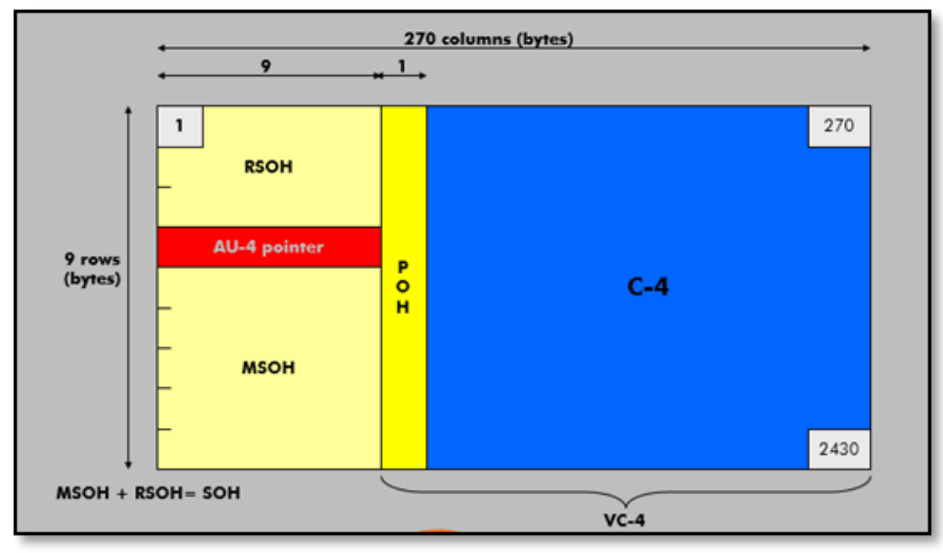

Figure 1: Frame format of STM-1 signal

It is also termed as VC-4 for STM-1 frame which is composed of path overhead(POH) and the actual payload known as Container. POH covers up 1 column and rest of 260 columns are taken up by the payload. The container contains the real data that is being carried over the network. When Path Overhead(POH) joins to the container, it forms a Virtual Container(VC). When we add pointer to VC it forms AU (Augmentation Unit). The main overhead contains MSOH and $\mathrm{RSOH}$.

\subsection{Section Overhead}

The section overhead further comprises-
1. $\mathrm{MSOH}$ (Multiplex Section Overhead)
2. RSOH (Regenerator Section Overhead)
3. Pointer

\section{$3.2 \mathrm{RSOH}$ (Regenerator Section Overhead)}

: RSOH is formed from the rows 1-3 in STM-1 frame in 9x9 section overhead. The physical connectivity between different equipment is well-defined in this section.

This section contains the following bytes-

- A1, A2 Bytes (Framing bytes)- There are three A1 and three A2 bytes in Regenerator Section used for framing pattern and which specifies the location from where STM-1 frame starts.

- C1 Byte (Channel Identifier byte)- $\mathrm{C} 1$ byte is channel identifier that is used to identify the STM-1 frames in higherlevel SDH frame where value of $\mathrm{N}$ for STM-N is 4,16,64 etc.

- B1 Byte (Error monitoring byte)- B1 byte is used to perform parity check in STM-1 frame. Thus, handles the error monitoring using 8-bit wide checksum.

- D1, D2, D3 Bytes (Data communication channel bytes)-These bytes are used as data communication channel between different regenerator section terminating equipment. 
- E1 Byte (Order wire bytes)- E1 byte provides an orderwire channel supporting voice communication between various regenerator section terminating equipment.

- F1 Byte (User communication byte)- This byte can be termed as channel used for user communication as it provides different channels terminating at regenerator section to the network operator.

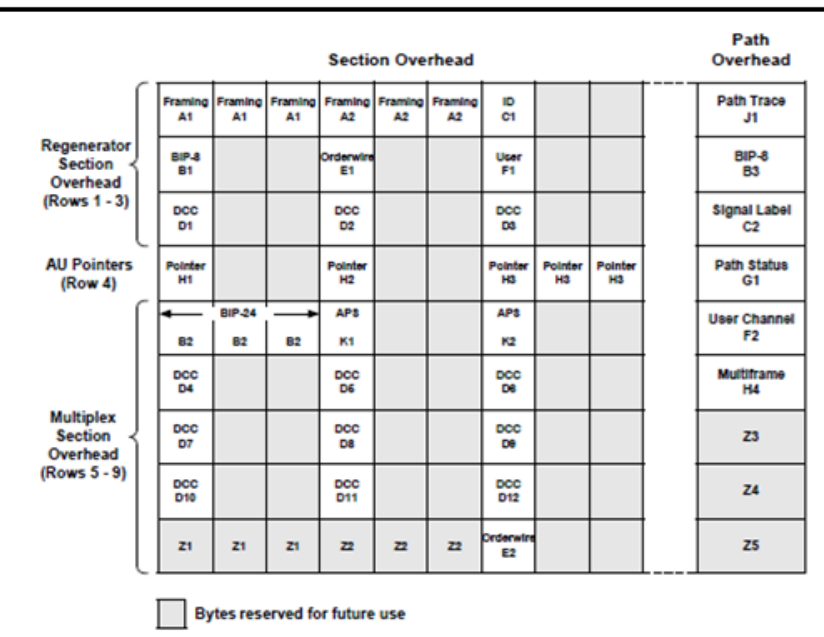

Figure 2: Section overhead and Path overhead bytes (Source: 28)

\subsection{MSOH (Multiplexer Section Overhead):}

The rows 5-9 in STM-1 frame in 9x9 SOH is termed as Multiplexer Section Overhead. This section handle all the multiplexing related activities in between adjacent network elements/equipment. This section can have up to three regenerator sections.

This section contains the following bytes-

- B2 Bytes (Error bytes)- These provide parity check over the bits of MS section and after performing Bit -interleaved parity check places the calculated checksum in MSOH section of the frame.

- K1, K2 Bytes (Switching bytes)- These are one of the significant bytes of STM frame as these are used for performing switching in main to protection paths and vice-versa so that there is a continuous flow of information in MS section.

- D4 to D12 Bytes (Data communication channel bytes)- These bytes are termed as data communication channel bytes as they provide data communication between different multiplexer terminating equipment and various maintenance services .

- E2 Byte (Order wire byte)- This byte provides an order wire channel supporting voice communication between various multiplexer section terminating equipment.

\subsection{AU-4 Pointer (H1, H2, H3 Bytes):}

AU-4 Pointer takes up one column in Section overhead comprising of H1, H2 and H3 bytes. AU-4links up section overhead with the virtual container.VC-4 can begin anywhere in an STM-1 frame to provide efficient multiplexing and better crossconnection of signals. Thus, pointers are required to mark the start point of payload data.

\section{AUTOMATIC PROTECTION SWITCHING(APS)}

It is a very important mechanism for network or link failure which involves switching of the traffic flow from main to the spare path having the capacity same as that of main path. With the increase in usage of data communication day by day if the network causes loss of data, it might shift the number of users/customers from one network to another. Therefore, protection has become an important part to maintain data flow throughout the network. APS involves the dependency on some of the criterion like the switching type is revertive/non-revertive and it is unidirectional/bidirectional. Synchronous multiplexing enables SDH to have ring topology which helps in sustaining single or multiple failures in the network. SDH frame has inbuilt provision to support protection mechanism. The STM frame's Multiplexer section(MSOH) has K1 K2 bytes for this purpose. 
The command type to activate protection is as follows-

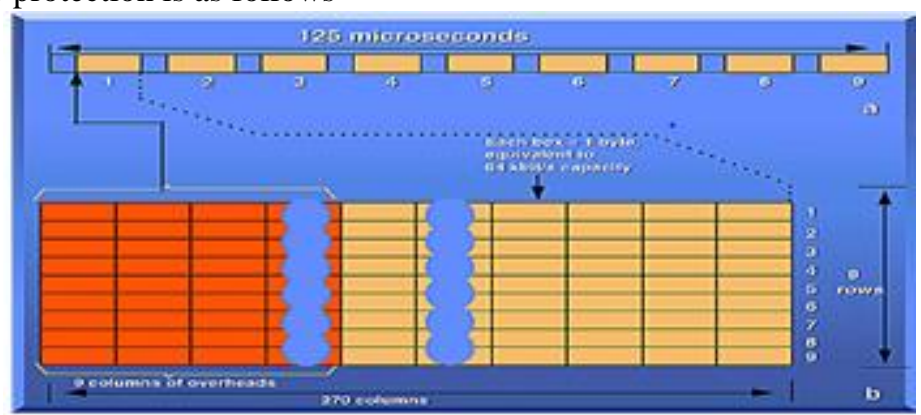

Figure 3: K1, K2 Bytes

\subsection{Automatically Initiated Conditions}

These conditions involve Signal Fail, Signal Degrade, Reverse Request and Wait to Restore. Signal Fail is the condition issued if there is no signal at receiver end network element. Signal degrade is the condition when the frame of SDH is distorted at receiver end network element. Reverse Request is the condition which is transmitted to tail end network element located on the short path to acknowledge receipt of short path ring request. Wait to restore is issued when working channels/ path meet the restorable threshold after signal fail/ degrade condition that is for two to ten minutes.

\subsection{Externally Initiated Conditions}

These commands involve manual switch, forced switch to protection, protection lockout or clear.

\section{PROTECTION IN SDH NETWORK}

The different protection types in SDH network is categorized as-
1.Line Protection
2. Path Protection

3. Ring Protection

\subsection{Line Protection}

Line Protection is based on the Linear APS or MSP principle. MSP is Multiplex Section Protection which involves two lines, one is termed as main line, another termed as protection line. Whenever there is fault in main line, the traffic is moved to the standby line and the flow of data through the protection line continues.

As seen in Figure 4, the blue line illustrates the main path and the other line red in color shows the protection path. For normal conditions traffic flows from main path. When failure occurs the traffic switches to protection path if the main path from node 1 to node 2 or vice- versa fails to transmit data.

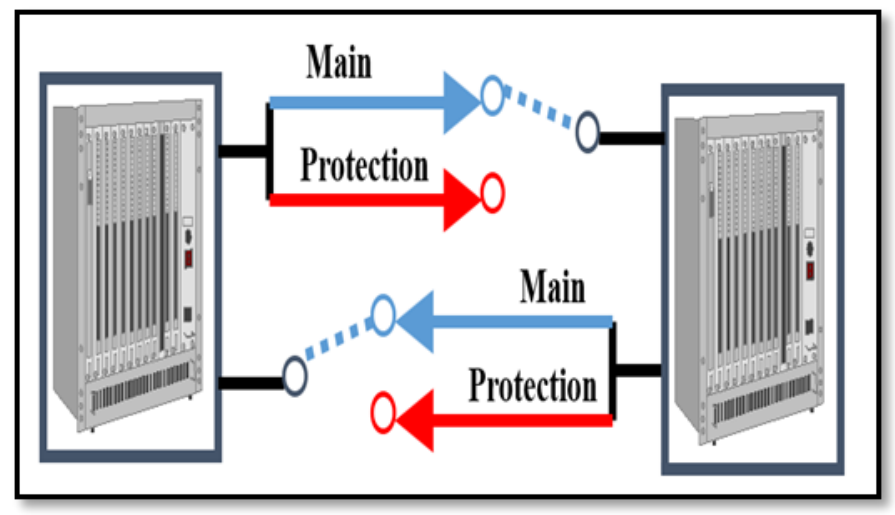

Figure 4: MSP Protection

\subsection{Path Protection (SNCP Protection)}

As we are discussing the product DXC MCC, it uses the protection feature SNCP (Subnetwork Connection Protection). In different $\mathrm{VC}$ levels whether it is $\mathrm{VC}-4$, VC-3 or $\mathrm{VC}-12$, this protection feature provides protection. 


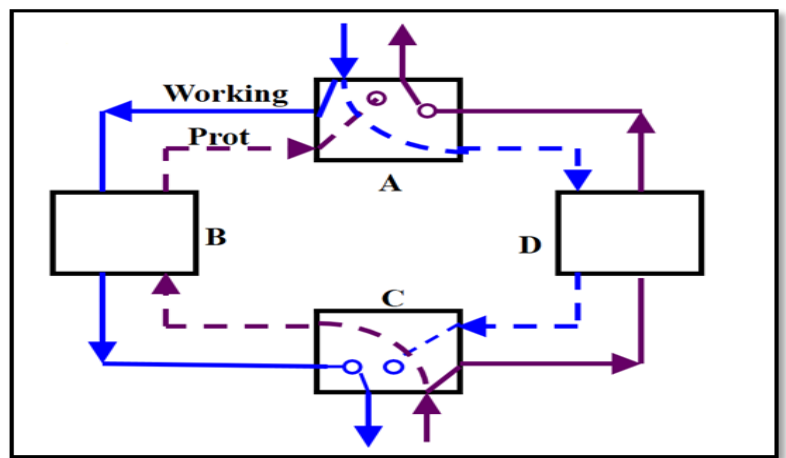

Figure 5: Ring in working condition

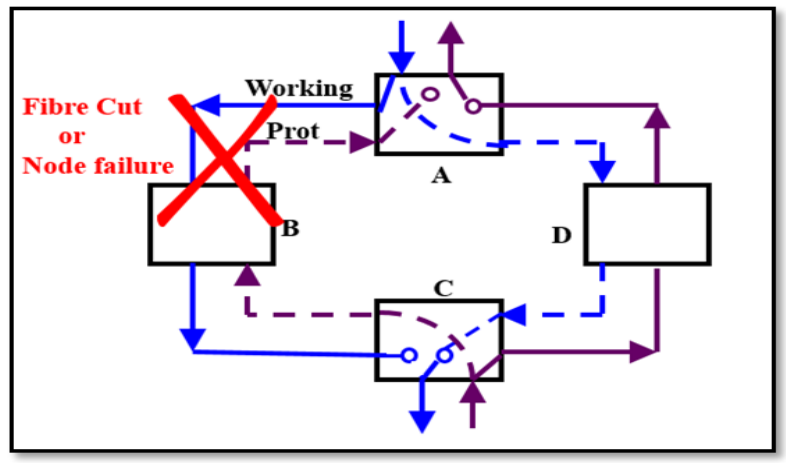

Figure 6: Ring in failure condition

In it transmission end is permanently bridged on main and spare paths. SNCP protection can be visualized in above figure where a ring is shown with four nodes. The blue line shows the main path connectivity between different nodes and violet line marks the spare path between different nodes. The movement of data frames normally can be seen in the above figure. In figure 6 we can visualize the movement of data frames when node fails in the ring and SNCP protection comes into play. The signal going from A to B and then from B to C. When there is media cut between A and B or issue in B node, the traffic signal shall shift automatically from A to D and then D to C(dropping Edge) and vice-versa making the protection active.

\subsection{Ring Protection (MSSPRing Protection)}

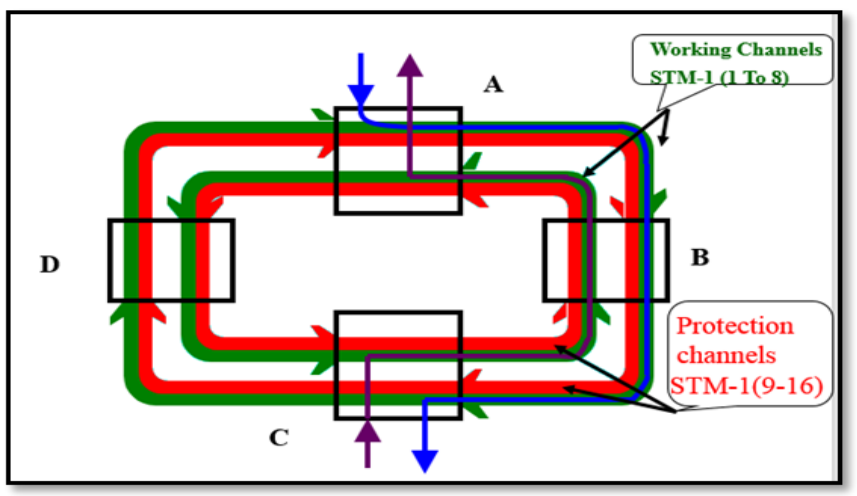

Figure 7: MS-SPRing in normal condition

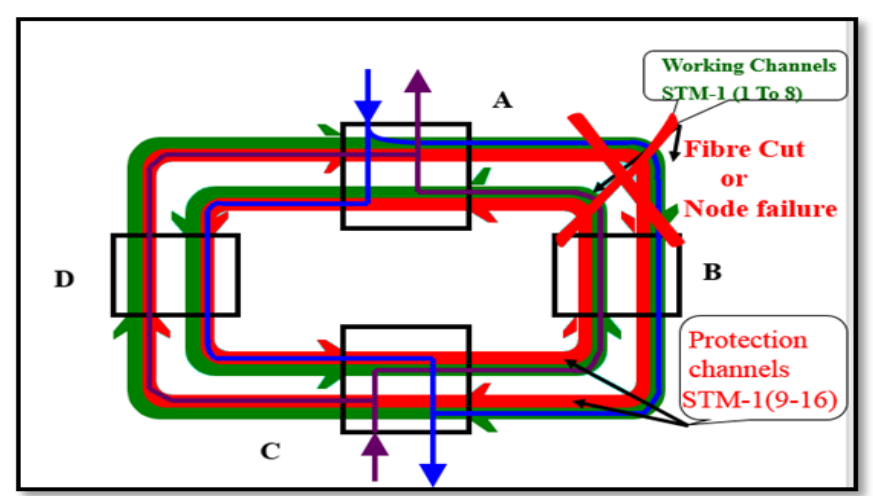

Figure 8: MS-SPRing in failure condition

The product involved in this case study uses the feature MS- SP Ring (Multiplex Section Shared Protection Ring). The two types of scheme used in ring protection are- $2 \mathrm{~F}$ or 2 Fibre and $4 \mathrm{~F}$ or 4 Fibre.

In $2 \mathrm{~F}$ ring, capacity of fibre is divided into two halves, out of which one handles the working traffic and another handles protection traffic whereas in $4 \mathrm{~F}$ ring, fibre's complete capacity is used for working traffic and $4 \mathrm{~F}$ ring has two protection fibre

two working fibres. In a two-fibre ring, the first optical fibre includes the first main channel and the protection channel and the second optical fibre is for second working channel and the protection channel. In a four-fibre ring, there are two pairs of bidirectional optical fibres. One pair works as two working channels and the second pair works as two protection channels. [29] MS-Spring supports dual ended and revertive switching in which the traffic switches back from protection path to the main path once the issue in main path resolves. In figure 7 we can visualize MS-Spring for normal scenario and figure 8 shows the MS-SPRing when node failure or fibre cut occurs.It can be noticed that half the capacity of STM frame is used as working channel and the second half as protection channel. For node failure in the transmitting end from A to C, switching takes place and protection gets activated and traffic continues to flow.

\subsection{Description Of Dxc}

The DXC (Digital Core Connect) is an intelligent node belonging to the family OMSN (Optical Multi-Service Nodes) which has density of $640 \mathrm{Gbit} / \mathrm{s}$ in a single shelf This network element is a smart device as it satisfies all the requirements providing an ideal transport solution. It helps in simplifying and optimizing the network, minimizing costs, enabling broadband services and easily supports future technologies like OTN (Optical Transport Network) architectures, lambda networking. SDH/ SONET are the two technologies which are nearly the same but their standards are defined by different institutes. ETSI (European Telecommunications Standards Institute) which is the subset of ITU-T (International Telecommunication Union- 
Telecommunication Sector) defines the SDH standard whereas ANSI (American National Standards Institute) defines the SONET (Synchronous Optical Networking) standard.

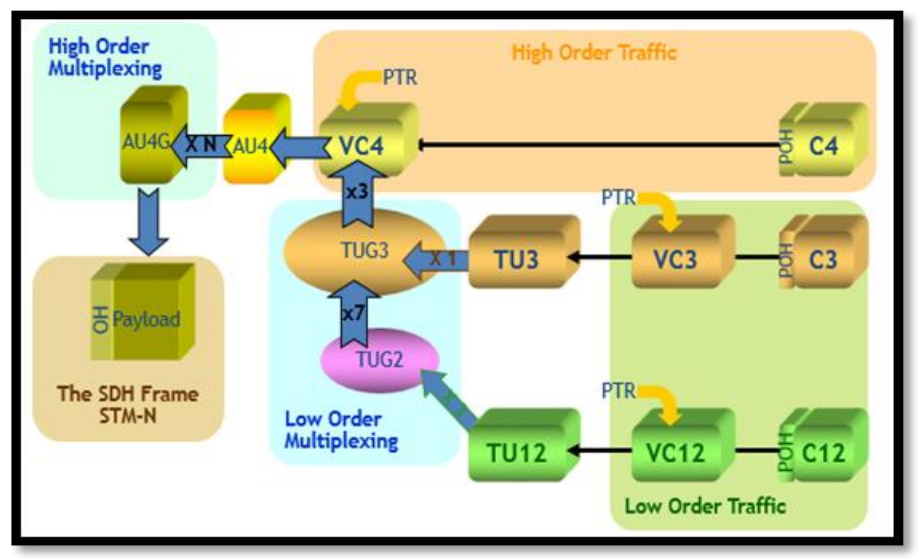

Figure 9: ETSI Multiplexing structure

SDH uses three containers as shown in figure 9 and rest others are used by SONET. The different components of SDH Multiplexing structure(ETSI) are

- Container: The basic frame component of the SDH frame is container that contains the actual data during transmission. It is like a 'box' that allows transport of information via PDH (Plesiochronous Digital Hierarchy) signals through SDH network. It is sized as per the data to be transmitted. 2 Mbps data is transmitted using container C12, 34 and 45 Mbps signals are transmitted using container C3 and 140 Mbps being the higher rate signal is transmitted using the container $\mathrm{C} 4$ as defined by ITU-T (International Telecommunication UnionTelecommunication Sector) recommendations.

- Virtual Container (VC): When Path Overhead(POH) is added to the container, it forms VC or Virtual Container. $\mathrm{VC}-\mathrm{n}=\mathrm{C}-\mathrm{n}+\mathrm{POH}$ where $\mathrm{n}$ varies as per the standard being used.

- $\quad T U$ (Tributary Unit): It is made up of a lower order VC-n $(\mathrm{n}=11,12,2,3)$ and a pointer. TU-n $=$ VC-n + PTR where $\mathrm{n}$ varies as per the standard being used.

- $\quad T U G$ (Tributary Unit Group): TUG-2/ TUG-3 consists of several types of capacity payloads with different sizes.

TUG-k=m *TU-n where $\mathrm{n}=11,12,2,3$

TUG- $2=4 *$ TU- 11 or $3 *$ TU- 12 or $1 *$ TU- 2

TUG-3 $=7 *$ TUG- 2 or $1 *$ TU-3

- $A U$ (Administrative Unit): It is made up of high order $\mathrm{VC}-\mathrm{n}(\mathrm{n}=3,4)$ and a pointer. AU-3 multiplexing structure is designed for compatibility with SONET frames. For SDH frames, AU-4s are used which further combine to form AUG(AU-group)

AU-n= VC-n + PTR when $n=3,4$

$\mathrm{AUG}=1 * \mathrm{AU}-4$ or $3 * \mathrm{AU}-3$

\section{DESCRIPTION OF VARIOUS SLOTS IN DXC}

In figure 10, different slots are shown. There are 16 port slots which support up to $40 \mathrm{Gbit} / \mathrm{s}$ throughout making up the total capacity of matrix $640 \mathrm{Gbit} / \mathrm{s}$.

There are two slots of control and common parts slot which contain the following boards-

$>$ First Level Controller and Service Interfaces (FLCSERV)

$>$ First Level Controller and Control \& General Interfaces (FLCCONGI)

$>$ Power Supply Filter (1+1) (PSF)

$>$ Bus Termination (two boards) (BUSTERM) 


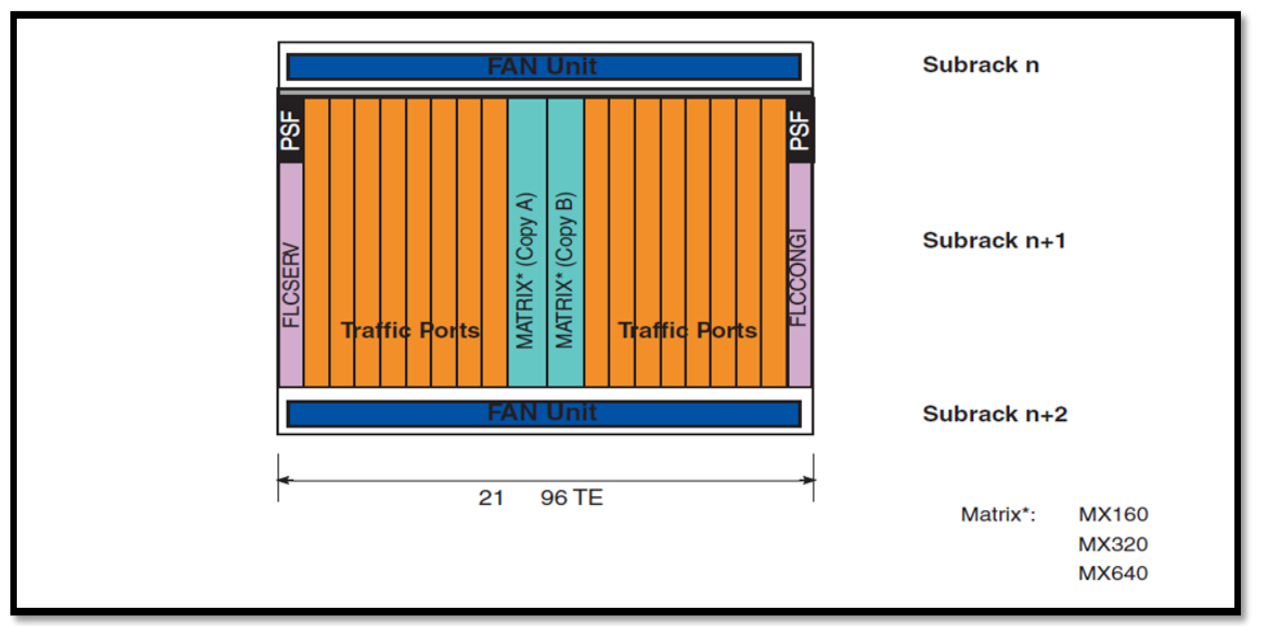

Figure 10: DXC Main Shelf Layout (Source: 30)

Table 2: DXC Slotwise card description

\begin{tabular}{|l|l|}
\hline Slot No. & Cards in the slot \\
\hline slot 1 & First Level Controller "spare" + Service Interfaces board (FLCSERV, FLCSERVA) \\
\hline slots 2 to 9 and slots 12 to 19 & $\begin{array}{l}\text { Traffic Port boards, ES64 data board, Lower Order Adaptation/Matrix and Lower } \\
\text { Order Matrix Link 40G }\end{array}$ \\
\hline slot 10 and 11 & $\begin{array}{l}\text { High Order Matrix (A and B) boards (MX640, MX640GA, MX320, MX320GA, } \\
\text { MX160) }\end{array}$ \\
\hline slot 20 & First Level Controller "main" + Control \&General Interfaces board (FLCCONGI) \\
\hline slot 21 and 22 & Bus Termination boards (BUSTERM) \\
\hline slot 24 and 25 & Power Supply and Filter boards (PSF) \\
\hline
\end{tabular}

It can be seen in figure 10 that there are two First Level Controllers or FLC's- FLCSERVICE and FLCCONGI

FLCSERVICE is the spare First Level Controller of the equipment. It is the flash memory device. It has an external synchronization interface along with $\mathrm{Q}$ and $\mathrm{F}$ interfaces.

FLCCONGI is the main First Level Controller of the equipment. It is also the flash memory device with external connectors for housekeeping, remote alarms, rack alarms and has Q and F interfaces and as DCC. The matrix boards support the functionalities; cross- connection, synchronization and shelf controller. The capacity of matrix boards are $160 \mathrm{~Gb} / 320$ $\mathrm{Gb} / 640 \mathrm{~Gb}$ which as per the number of cross- connections to be handled by the equipment. The PSF (Power Supply Filter) board provides the distribution of power to the equipment after filtering process. The BUSTERM (Bus Termination Board) provides electrical termination to the buses routed on the backplane. The two BUSTERM are placed behind FLCSERVICE and FLCCONGI. The Fan unit has the functionality to provide cooling of the equipment. The two fan units are physically integrated in the lower order extension shelf, without the need of external connection cables. The two fan units are always to be equipped with the device due to its high level of integration and as it handles many cards so it is very important to maintain its temperature.

\section{CPE ALARM CASE STUDY}

The alarm described in this case study is CPE (Communication Protocol error). The alarm occurs when the K1/ K2 bytes of MS section violate the rules defined for the protocol for switching protection. The effect on service is that the protection does not operate properly and the automatic switching requests and operator commands for switching does not work. Therefore, the switching operation to the protection path does not take place in the case of failure in main path which affects the traffic that is supposed to flow from the protection path. The root cause analysis report is shared in this case that describes the issue. Figure 11 shows the MS-SPRing in which the issue was reported in the 2016-year end. The detailed description of the issue is given below.

\subsection{Background:}

It was informed by the customer that CPE issue is reflecting on node A s112 P04 and node B s112 p03in MS-SPRing Ring A. While investigation, it was found that there is media cut between A ---B Section, whereas CPE alarm was reflecting on node A sl12 P04 and node B s112 p03, due to which traffic was impacting. Issue was taken up with backend team, where it was found that intermediate node C s112p04 K2 switching was in hang-up state. S112 reset was performed, after that issue resolved. Further sl12 was replaced for permanent resolution of the issue. 


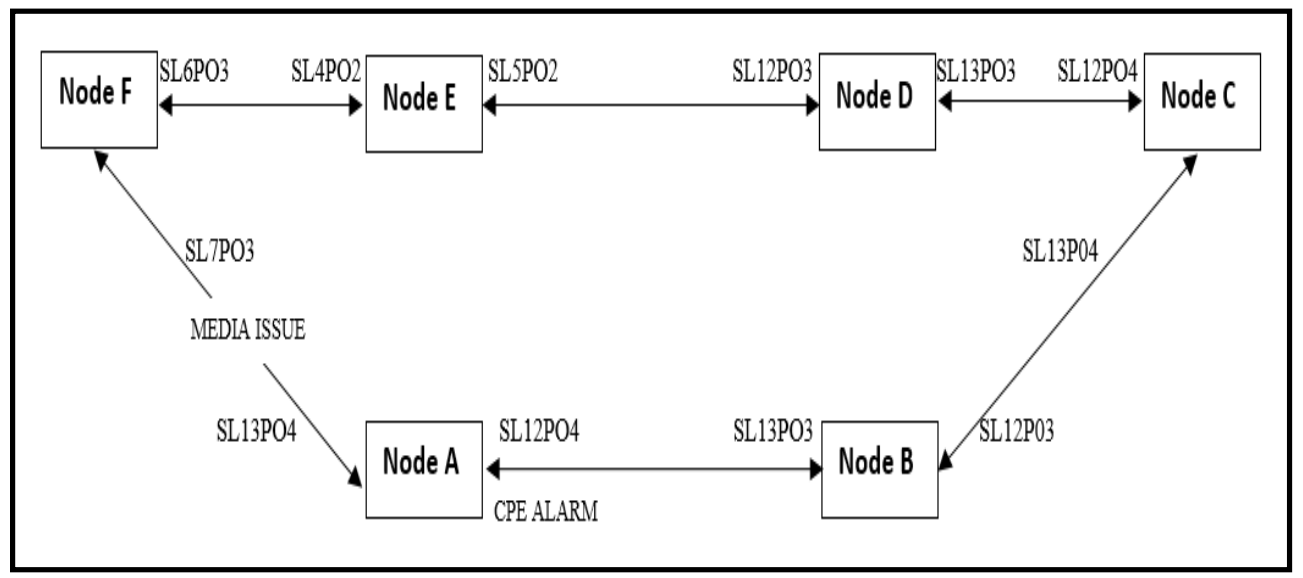

Figure 11: Connectivity of different nodes in MS-SPRing Ring A

\subsection{6-DEC-2016 11:32 AM:}

The issue was reported by the customer on this date reporting the CPE alarm in the node A sl12P04(slot 12, port 4) in MSSPRing Ring A.

\subsection{6-DEC-2016 11:52 AM:}

We checked and found there was fiber cut in Ring A between node A and node F and in the same duration CPE alarm reported at 1-3-12-4 of node A at another side of the node A(s112P04)----node B(s113P03) and node B (sl12p03) -----node C Link (shown in Figure 11). To check for the alarm CPE, we need to check the alarm logs that would clarify the time and date of the occurrence of alarm on specified slot and port. In Nokia, there is the use of Optical Management System to find out the alarm logs. After following the procedure to find alarms, the alarm logs as shown in Figure 12 were found.

\begin{tabular}{|c|c|c|c|c||}
\hline Event Date and Time & Node Name & Slot & Port & Probable Cause (name) \\
\hline $12 / 26 / 201611: 04$ & Node B & r01sr3sl12 & port\#03-MsTTP & Communication Protocol Error \\
\hline $12 / 26 / 201611: 04$ & Node A & r01sr3sl12 & port\#04-MsTTP & Communication Protocol Error \\
\hline $12 / 26 / 201611: 46$ & Node B & r01sr3sl12 & port\#03-MsTTP & Communication Protocol Error \\
\hline $12 / 26 / 201611: 46$ & Node A & r01sr3sl12 & port\#04-MsTTP & Communication Protocol Error \\
\hline $12 / 26 / 201612: 04$ & Node A & r01sr3sl12 & port\#04-MsTTP & Communication Protocol Error \\
\hline $12 / 26 / 201612: 18$ & Node A & r01sr3sl12 & port\#04-MsTPP & Communication Protocol Error \\
\hline $12 / 26 / 201613: 01$ & Node B & r01sr3sl12 & port\#03-MsTPP & Communication Protocol Error \\
\hline $12 / 26 / 201613: 01$ & Node A & r01sr3sl12 & port\#04-MsTTP & Communication Protocol Error \\
\hline $12 / 26 / 201614: 04$ & Node B & r01sr3sl12 & port\#03-MsTTP & Communication Protocol Error \\
\hline $12 / 26 / 201614: 04$ & Node A & r01sr3sl12 & port\#04-MsTTP & Communication Protocol Error \\
\hline
\end{tabular}

Figure 12: Alarm logs showing the occurrence of CPE alarm

\subsection{6-DEC-2016 12:25PM:}

While prelim diagnosis warm reset (remotely) was performed on both end node A s112 and node B slot 13, but issue still existed. After some duration media issue got resolved between A---B link and CPE alarm got auto cleared in MS-SPRing Ring A. Case got escalated to backend and but since CPE found cleared in MS-SPRing and got difficult to diagnose in case of MS-SPRing in normal state, as K2 byte value needs to be calculated during live scenario, it was requested to perform MSSPRing testing in maintenance window to diagnose the issue.

\subsection{7-DEC-2016:}

Lots of media issue (media issue involves fibre cut or any patch cord issue or any cable related issue due to which the signal does not flow in the cable between the section where media issue occurs) reported and found on $27 \mathrm{dec}$ due to which CPE reported multiple times and for some duration dual media issue reported in the ring. There is the alarm named LOF (Loss of Frame) that shows that frames are being lost between node A and node F due to which the traffic was to flow through node D but dual media issue occurred between node $\mathrm{D}$ and node $\mathrm{C}$ that 


\begin{tabular}{|c|c|c|c|c|}
\hline Event Date and Time & Node Name & Slot & Port & Probable Cause (name) \\
\hline $12 / 26 / 201611: 04$ & Node A & r01sr3s/13 & port\#03-0pRsCTP & Loss Of Frame \\
\hline $12 / 26 / 201613: 13$ & Node A & r01sr3s/12 & port\#04-0pRsCTP & Loss Of Frame \\
\hline $12 / 26 / 201611: 00$ & Node F & r01sr3s/07 & port\#04-0pRsCTP & Loss Of Frame \\
\hline $12 / 26 / 201613: 13$ & Node B & r01sr3s/13 & daughter\#03 & Replaceable Unit Missing \\
\hline $12 / 26 / 201613: 13$ & Node B & r01sr3 & board\#13 & Replaceable Unit Problem \\
\hline \hline
\end{tabular}

Figure 13: Alarm logs showing LOF (Loss of Frame) alarm observed on Port 4 of node F stopped the traffic flow and caused 7.6 28th Dec-16 3:00 AM: outage.

Field engineer reached at node A and node F site, where node A sl12 and node B sl12replaced, but CPE alarm still not cleared. meanwhile dual media cut happened in same ring (D---C section), issue couldn't be concluded. Later, node A field engineer was not available for further activity due to bulk media cut in the customer network.

\subsection{8th Dec-16 6:00 AM:}

The field engineer was available at both side, where A---B WDM section port shifted, but no change observed. Issue was taken up with upper level Team, where it was found that intermediate node $\mathrm{C}$ sl12 created problem by hanging K2 byte status for switching the MS-SPRing, due to which CPE alarm was reflecting on trailing node A and node B. Issue resolved after resetting slot12 P4S64X card of the node C. Node C slot 12 card replaced, under Maintenance Window for permanent resolution.

\subsection{Root cause Analysis:}

It was identified that when there was media cut in A---B Section or in A---F link the traffic expected to shift on protection AU\#4 in MS-SPRing Ring A, but in intermediate node Cslot12p04 switching byte couldn't respond to trigger protection switching. It was found that issue occurred due to hardware problem issue on card which was responsible to handle the K1K2 protection bytes. When the neighbor node receives this $\mathrm{K} 1 \mathrm{~K} 2$, there is computational error in the APS. Hence, CPE alarm occurred on the neighbor node.

\section{CONCLUSION AND DISCUSSION}

There are rare circumstances when such incidents would occur that are responsible for causing traffic outage. As the MSSPRing had another path to follow up in case of media issue but it was also affected by hardware failure which was later discovered when network analysis was carried out. The CPE issue appeared due to transit hardware failure appeared in node C S112P04, as K1K2 switching byte status couldn't be changed after media cut appeared in MS-SPRing. Such issue can be resolved at the same time after remote reset performed to the affected card. There should be provisions available that would help in advance checking of the cards at different critical nodes to prevent such cases to re-happen.

\section{ACKNOWLEDGEMENT:}

This case study was supported by Alcatel-Lucent (A Nokia Corporation Company). The product described and used in the case study is its proprietary. We express our gratitude to the corporation and the team members who assisted us in preparation of the manuscript.

\section{REFERENCES:}

[1] 1.Ferguson, S. P. "Implications of SONET and SDH." Electronics \& communication engineering journal 6.3 (1994): $133-142$.

[2] 2 Bregni,Stefano."A historical perspective on telecommunications network synchronization." IEEE Communications Magazine 36.6 (1998):158-166.

[3] MA'ITHEWS, M., and NEWCOMBE, P.: The synchronous digital hierarchy', ZEE Review, May 1991, pp.185-189, and June 1991, pp.229 - 233

[4] 4.Compton, Mark, and Simon Martin. "Realising the Benefits of SDH Technology for the Delivery of Services in the Access Network." Communications, 1994. ICC'94, SUPERCOMM/ICC'94, Conference Record,'Serving Humanity Through Communications. 'IEEE International Conference on. IEEE, 1994.

[5] 5. Rados, Ivan, Tanja Sunaric, and Per Turalija. "Availability comparison of different protection mechanisms in SDH ring network." Modern Problems of Radio Engineering, Telecommunications and Computer Science, 2002. Proceedings of the International Conference. IEEE, 2002.

[6] 6. Draft proposed American National Standard SONET Automatic Protection Switching contained in ANSI TIX1.5/93-057.

[7] 7. Bellcore Technical Adversory, TA-NW-000253, Synchronous Optical Networks (SONET) Fiber Optic Transmission Systems Requirements and Objectives, issue 6, Sep. 1990.

[8] 8. Klymash, Mychailo, et al. "Stream optimization of SDH networks with reservation." Modern Problems of Radio Engineering, Telecommunications and Computer Science, 2004. Proceedings of the International Conference. IEEE, 2004.

[9] 9. Weippert, Werner. "The evolution of the access network in Germany." IEEE Communications Magazine 32.2 (1994): $50-55$. 
[10] 10. Antonopoulos, Andreas, J. J. o 'Reilly, and Peter Lane. "A framework for the availability assessment of SDH transport networks." Computers and Communications, 1997. Proceedings., Second IEEE Symposium on. IEEE, 1997.

[11] 11.“Configuring Redundancy for POS APS," Cisco White Paper, June 2002, http://www.cisco.com/warp/public/127/POS/pos-aps_14680.pdf

[12] 12. Veitch, Paul. "A survivable and cost-effective IP metro interconnect architecture." IEEE Communications Magazine 41.12 (2003): 100-105.

[13] 13. Ueda, Hiromi, Hisao Tsuji, and Tosh nori Tsuboi. "New synchronous digital transmission system with network node interface." Global Telecommunications Conference and Exhibition' Communications Technology for the 1990s and Beyond'(GLOBECOM), 1989. IEEE. IEEE, 1989.

[14] 14. Y. Yamabayashi, et al., "Very high speed optical line terminals", IEEE GLOBECOM 199 1, pp. 22.5 .1 - 22.5.5

[15] 15. Xiao-Ping, Yan, and Ye Pei-Da. "Survivability study of SDH self-healing ring." Communication Technology Proceedings, 1996. ICCT'96., 1996 International Conference on. Vol. 2. IEEE, 1996.

[16] 16. Yongming, Xu, Zhang Xiaopin, and Ye Peida. "Asynchronous mapping of 2.048 Mbit/s tributary into SDH VC-12." Communication Technology Proceedings, 1996. ICCT'96., 1996 International Conference on. Vol. 2. IEEE, 1996.

[17] 17. A. McGuire, P. Bonenfant, "Standards: The Blueprints for Optical Networking", IEEE Comm. Magazine, vol. 36, pp. 68-78, 1998.

[18] 18. Cheng, Joe Z., Peng-Ting Chen, and D. Yu Hsiao-Cheng. "Establishing a MAN access strategy for future broadband service: a fuzzy MCDM analysis of SONET/SDH and Gigabit Ethernet." Technovation 25.5 (2005): 557-567.

[19] 19. Kim, Si-Won, and Ki-Shick Park. "Functional considerations for development of SDH technology-based broadband digital cross-connect system." Computers and Communications, 1998. ISCC'98. Proceedings. Third IEEE Symposium on. IEEE, 1998.

[20] 20. ITU-T Rec. G.841, Types and characteristics of SDH network protection architectures, 1998.

[21] 21. M. To, P. Neusy, Unavailability analysis of long-haul networks, IEEE Journal on Selected Areas in Communication 12 (1994) $100-109$.

[22] 22. J.-P. Vasseur, M. Pickavet, P. Demeester, Network Recovery - Protection and Restoration of Optical, SONET-SDH, IP and MPLS, Elsevier, San Francisco, 2004.

[23] 23. Zalevsky, Z., and R. Appelman. "All-optical switching technologies for protection applications." IEEE Communications Magazine 42.11 (2004): S35-S40.

[24] 24. Johnson, Dave, Nigel Hayman, and Paul Veitch. "The evolution of a reliable transport network." IEEE Communications Magazine 37.8 (1999): $52-$ 57.

[25] 25 Veitch, P. A. "Alternative transport architecture for core ATM networks." BT technology journal 16.3 (1998): 205-211.

[26] 26.Veitch, Paul, and Dave Johnson. "ATM network resilience." IEEE Network 11.5 (1997): 26-33

[27] 27. "Introduction to the Synchronous Digital Hierarchy (SDH)" http://mars.merhot.dk/mediawiki/images/d/d0/Introduction-to-SDH.pdf

[28] 28."SDH/SONET Environment-RAD USA" http://www.radusa.com/Media/6971_SDH_SONETbackgrounder.pdf

[29] 29. "SDH ring high order path management" https://www.google.ch/patents/US5815490

[30] 30. Alcatel Lucent proprietary: DXC product and planning guide 